\title{
Sur les coefficients des fonctions exponentielles reliant les débits maxima des crues aux aires des bassins versants
}

\section{Exponential coefficients used to express maximum flood discharges as functions of the areas of drainage basins}

\author{
PAR M.-M. CHARTIER \\ PROFESSEUR IE LYCÉE DÉTACHÉ AU C.N.R.S. \\ Rapport élaboré par M. Marcel-M. Chartier, d'après un important mémoire de M. Maurice PARDE

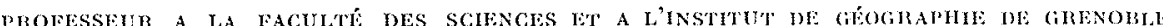

Il esl notoire qu'en règle générale les débits maxima @ des crues de même fréquence augmentent moins que les surfaces réceptrices $\mathrm{S}$. On a voula lier $\mathrm{Q} \dot{a} \vee \widehat{S}$ par des coefficients $\mathrm{A}=\mathrm{Q} \vee \overrightarrow{\mathrm{S}}$, puis par des coefficients $\mathrm{A}^{\prime}=\mathrm{Q} / \mathrm{S}^{2 / 3}$ ou par des paramètres similaires, associés d̀ des exposants aulres que $1 / 2$ ou $2 / 3$ des silrfaces réceptrices.

Alucun de ces coefficients ne pouvait avoir d'exactilude universelle, dans un monde oil les facteurs géphysiques des crues offrenl une extrême variété. Mais il existe des régions, on des sous-régions, géphysiquement assez homogènes pour que les crues. d'une fréguence donnée (et même les débits records connus) soient caractérisés par certains jeux de valeurstypes pour les coefficients $\mathrm{A}, \mathrm{A}^{\prime}$, etc., entre certains chiffres limites des surfaces réceptrices: par exemple, on trouve pour $A$ record, 10 a 12 dans le bassin de la Seine, 40 d 50 pour la Dordogne, la Durance d̀ Mircibeatu, le Lot, plas pour le Turn, la Garonne da Toulouse, 100 d̀ 175 pour plusieurs rivières cévenoles. Dans cette communication présentée a la S.H.F. et qui résume un mémoire considérable, M. Maurice Pardé examine ces valeurs, les facteurs qui les déterminent, et les causes d'exceptions. Cette etude doit envisager tout un monde très complexe et nuancé de phénomènes.
It is well known that, generally speaking, the maximum discharges $\mathrm{Q}$ of floods occurring witi the same frequency do not increase as fast as the areas $S$ of the associated drainage basins. Attemps were made to express $\mathrm{Q}$ as a function of $\sqrt{\mathrm{S}}$ by means of coefficients of the type $A=Q \sqrt{\mathrm{S}}$, and later by coeffcients of the type $A^{\prime}=0 / \mathrm{S}^{2 / 3}$, or with similar parameters associated with exponentials of the areas other than $1 / 2$ or $2 / 3$.

No one coefficient could be accurate in all cases when the geophysical aspects of floods are so extremely varied. But regions or sub-regions exist which have sufficient geophysical homogeneity to make it possible to associate floods of a given frequency, and this include even record floods, with sets of typical values of the coefficients $A, A^{\prime}$, etc., cerlain limits being set upon the areas of the drainage basins. Record balues of A are: 10 to 12 for the Seine Basin, 40 to 50 for the Dordogne, for the Durance at Mirabeat, for the Lot and even higher for the Tarn and for the Garonne at Toulouse, with 100 to 175 for certain rivers in the Cevennes. In this paper which is a summary of a more extensive work, the authors examine such values together with the factors that govern them and the causes of the exceptions that occur. The work described in this paper involved considering a very complex and very finely graduated body of natural phenomena. 
Depuis quelques dizaines d'années à peine, le matériel numérique relatif à l'Hydrologie fluvial s'est immensément accru. Les progrès considérables de l'Hydrométrie apportent constamment des données nouvelles, voire des révélations et des éléments de rectification. Ainsi, les hydrologues ont-ils pris pleine conscience du fait qu'en beaucoup de régions, selon une évolution qu'on peut qualifier de normale, les débitsrecords, exprimés en débits maxima bruts $\left(\mathrm{m}^{3} / \mathrm{s}\right.$ ou pieds cubes/seconde), n'augmentent pas en fonction linéaire des surfaces réceptrices, mais en fonction de puissances de celles-ci inférieures à l'unité (1). On conçoit alors que de nombreux auteurs aient cherché une formule capable de fournir un critère facilement accessible permettant d'apprécier n'importe quel débit maximum émanant de n'importe quelle surface.

La plus simple de ces formules, peut-être la moins défectueuse, a été proposée originelle- ment semble-t-il, par Myer, puis recommandée ultérieurement par l'Hydrologue réputé, A. Coutagne. Elle exprime que le débit maximum augmente en fonction d'une puissance de la surface du bassin voisine de $1 / 2: Q=A \sqrt{S}(Q=d e ́-$ bit brut maximum en $\mathrm{m}^{3} / \mathrm{s} ; \mathrm{S}=$ surface réceptrice en $\mathrm{km}^{2}$; $A=$ coefficient variant régionalement selon les facteurs géophysiques, mais qu'on espère stable de l'amont à l'aval pour certains grands bassins, jusqu'à 50000 ou $100000 \mathrm{~km}^{2}$ et même plus, ou pour certains groupes de bassins dans une région homogène). C'est une sélection de nombreuses valeur's effectives du coefficient $A$ et une comparaison des puissances respectives des débits maxima des crues connues en maintes parties du globe qui sont présentées dans cette communication, d'après un énorme mèmoire de M. Maurice Pardé qui a, une fois de plus, mis son encyclopédique documentation au service de son esprit critique.

\section{EXISTENCE DE VALEURS-TYPES REGIONALES POUR A}

Pour des surfaces réceptrices d'étendues petites ou modérées, comprises généralement entre 200 et $20000 \mathrm{~km}^{2}$, parfois un peu plus, d'abondantes données sérieuses montrent que, sans avoir à l'intérieur de chaque région, à défaut d'une uniformité absolue impensable, une stabilité relative très satisfaisante, A offre des jeux de valeurs-types qui constituent pour ces domaines terrestres des indices géographiques importants et significatifs. Toute anomalie suggère des causes à bien déterminer.

En France, abstraction faite de certains secteurs où sévissent les déluges méditerranéens, il est rare que les valeurs de A soient supérieures à 60 . Sur la Seine, on ne peut attendre de fortes valeurs de $A$ en raison de la modération des averses et de la faiblesse générale des pentes, sauf sur l'étroit secteur du Morvan :

\begin{tabular}{|c|c|c|}
\hline Seine & $\begin{array}{c}\text { Amont } \\
\text { du confluent } \\
\text { avec l'Yonne }\end{array}$ & $\begin{array}{c}\text { Paris } \\
\text { (Austerlitz) }\end{array}$ \\
\hline Janvier 1910. & 6,4 & 11,2 \\
Janvier 1955. & 6,9 & 9,8 \\
Janvier 1959. & 6,0 & 8,6 \\
\hline
\end{tabular}

Sur la Loire, l'indice diminue de la source

(1) Ce qui implique une diminution corrélative exponentielle des maxima spécifiques en litres-seconde par $\mathrm{km}^{2}$ ou en pieds cubes-seconde $\left(28,8 \mathrm{dm}^{3}\right)$ par mille carré $\left(2,59 \mathrm{~km}^{2}\right)$. jusqu'à l'embouchure, de façon presque continue à partir du Bec d'Allier (où $A$ varie de 47 à 52) jusqu'à Montjean où les crues-records ne nous donnent que $A=20$ ou 21 , tandis qu'à Tours, nous trouvons encore $\mathrm{A}=\mathrm{de} \quad 30$ à 34 .

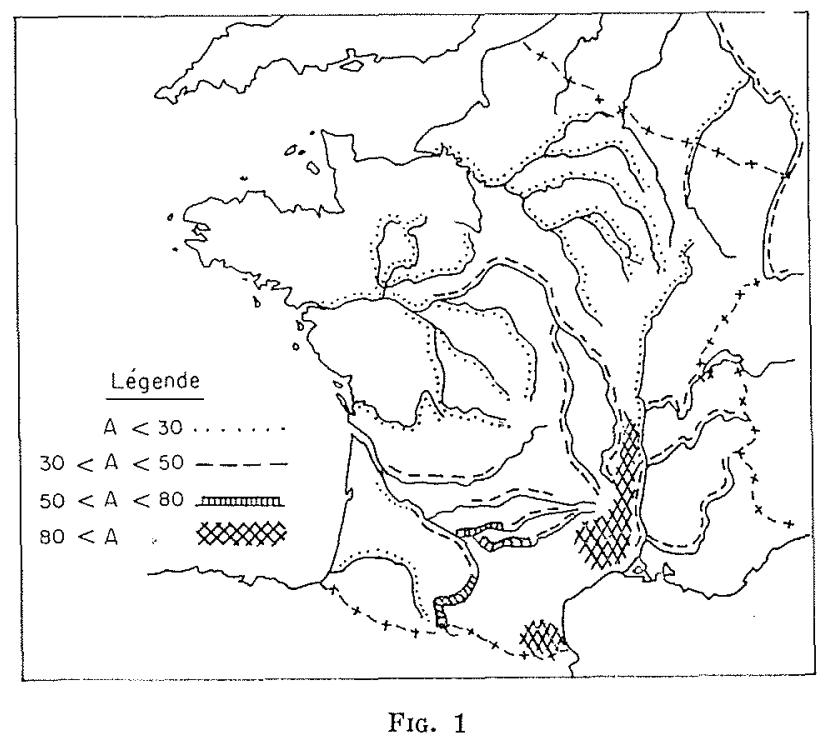

Valeurs de $A=Q^{/ S^{1 / 2}}$ pour quelques cours d'eau français.

Sur le Rhône, compte non tenu de la partie suisse qui aboutit au lac Léman, A vaut 40 en amont du confluent avec la Saône et moins de 30 à Givors, en aval; mais A remonte, notamment à partir du confluent avec l'Isère, pour 
atteindre 35 à Valence et de 37 à 40 à Beaucaire. Quant à la Garonne, dont le bassin éprouve de fortes crues, les valeurs de A décroissent notablement en aval du Lot, en dépit de la convergence morphologique et à cause de graves submersions qui favorisent l'aplatissement des maxima bruts. De 70-75 à Toulouse $(60-65$ à Montauban), A passe à 43-48 à Agen, 30-35 a Tonneins et 25-30 vers La Réole et Langon. En résumé, les coefficients A des principales rivières francaises (abstraction faite des crues cévenoles, languedociennes ou roussillonnaises) varient dans un rapport de 1 à 10 ou 11. De plus, ils soulignent le fait, commun à bien des bassins fluviaux, que les débits maxima bruts non seulement n'augmentent pas aussi vite que les surfaces réceptrices, mais encore peuvent diminuer de l'amont vers l'aval par suite du phénomène de l'aplatissement.

En Europe, toujours en dehors des régions méditerranéennes, pour les bassins d'étendue modérée comme pour les grands bassins, les valeurs de $\mathrm{A}$ demeurent généralement plus faibles que 50: elles n'impliquent guère plus de 10 pour l'Elbe et l'Oder inférieurs et atteignent 18 à 25 à l'issue des bassins moyennement accidentés et arrosés comme eeux de la Moselle, du Neckar ou du Main, 25 à 30 pour le Rhin et pour le Danube bavarois, 35 à 50 pour le Danube autrichien, pour plusieurs branches de la Vistule supérieure, pour les cours inférieurs du Pô, du Douro et du Tage, 60 et plus pour le Pó moyen à Piacenza. Pour les fleuves ibériques, les maxima s'aggravent en puissance relative d'amont en aval, car les rios échappent à la Meseta peu arrosée et atteignent, puis tra- versent, des régions frappées par des pluies sévères : le coefficient $A$ monte le long du Douro de 17,1 (amont) à 45 ou 48 (aval). La même constatation est faite avec les fleuves de la Russie d'Europe (et de la Sibérie), mais à cause de la liquéfaction, en une seule phase prolonsée, d'une couche de neige couvrant plusieurs centaines de milliers de $\mathrm{km}^{2}$ d'un sol préalablement gelé ou ultra-saturé : A croît donc en fonction des surfaces réceptrices de 31,4 à 61,3 pour la Petchora, de 19,2 à 40 pour le Dniepr, de 24 à 61 pour la Volga, de 34 à 76 pour l'Iénisséi et de 37 à 71 pour la Léna.

Avec les régions méditerranéennes de l'Europe O'ccidentale, du Roussillon et des Cévennes à la Calabre et à la Sardaigne, les coefficients A connaissent, à cause de reliefs propices aux ruissellements rapides et surtout de pluies torrentielles, des valeurs bien plus considérables; les débits maxima des crues formidables et foudroyantes paraissent particulièrement plus impressionnants, même, que les volumes totaux souvent modérés, mais sont corroborés par de remarquables débits spécifiques, lorsqu'il s'agit de très petits bassins; d'ailleurs, les phénomènes sont différenciés tant par la virulence de l'averse que par la morphologie de la nature du sol. C'est ainsi que l'Oued Hammam, à Bou Hanifia, dans l'Oranais, a atteint au plus 56,5 pour son maximum connu (novembre 1927) à l'issue de $7800 \mathrm{~km}^{2}$, cependant que le Jucar espagnol accuse probablement 90 à l'issue de $17000 \mathrm{~km}^{2}$, la Drina yougoslave, 95 pour $11000 \mathrm{~km}^{2}$, les rivières françaises roussillonnaises et cévenoles, de 90 à 150 (Ardèche) et peutètre plus (175 pour le Tech en octobre 1940?).

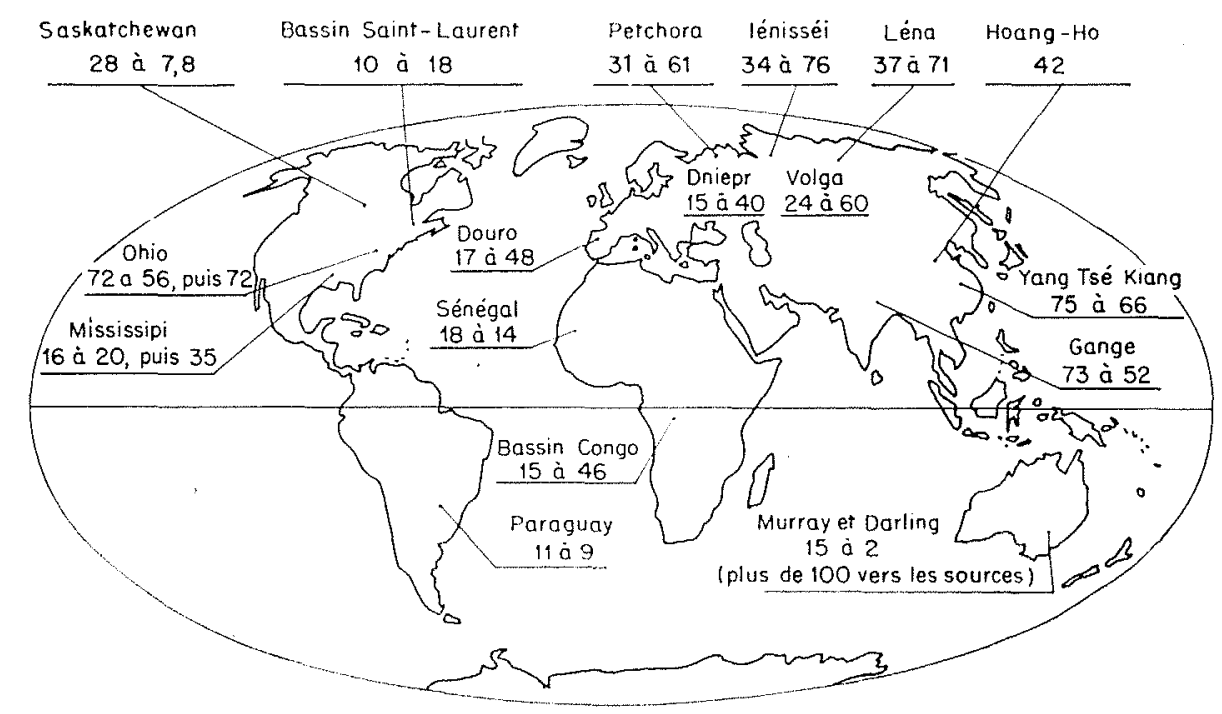

Fig. 2

Valeurs de $A=Q / S^{1 / 2}$ pour divers fleuves du monde à quelques stations. (Les valeurs de A sont eitées de l'amont vers l'aval.) 
Sur le haul Flumendosa sarde enfin, on note 133 en octobre 1951.

Toutefois, les résultats de ces intempéries pluviales méditerranéennes sont nettement dépassés par les valeurs rencontrées aux EtatsUnis d'Amérique du Nord et au Japon : dans Ie Nebraska, 268; au Texas, plus de 300; au Japon, de 200 à 250 pour des bassins couvrant jusqu'à $5000 \mathrm{~km}^{2}$.

Ces nombreux exemples montrent l'intérêt du coefficient $A$, sa signification géographique. D'après ses valeurs, l'Afrique Noire inter-tropicale présente des crues qui n'ont pas, à beaucoup près, la violence que trop souvent on leur suppose, aussi bien pour les petits bassins que pour les bassins d'étendues moyennes ou grandes; il nous semble exceptionnel que le coefficient $A$ puisse dépasser 25 à 30 pour 10000 à $20000 \mathrm{~km}^{2}$. Mais, à l'issue de bassins minus- cules, les crues peuvent être très fortes quoique sans doute moitié moindres qu'aux Etats-Unis. Puis, les chiffres sérieux que nous possédons pour une partie restreinte de l'Amérique du Sud, le centre méridional du Brésil, donnent l'impression qu'à l'issue de surfaces réceptrices de 5000 à $25000 \mathrm{~km}^{2}$, A reste en général inférieur à 25. Au sud (Uruguay, Jacui, etc.), il peut être bien plus élevé.

En somme, le coefficient A peut, dans certains cas, avec un choix judicieux, permettre d'évaluer très vite, au moins provisoirement et grossièrement, avec une assez bonne sécurité, certains très forts débits à craindre ou à utiliser sans qu'il soit nécessaire de recourir à des méthodes plus précises, mais plus longues. Toutefois, cette formule n'est pas pleinement satisfaisante et conduit à des inexactitudes, voire à des échecs.

\section{INSUFFISANCE DE L'EXPOSANT $1 / 2$}

Des défectuosités sérieuses du coefficicnt $A=Q \sqrt{S}$ apparaissent au' débouché de bassins exigus (moins de 100 ou de $50 \mathrm{~km}^{2}$ ) et au-dessus de surfaces réceptrices-limites approximativement voisines de $5000 \mathrm{~km}^{2}$ ou peut-être de $10000 \mathrm{~km}^{22}$; le plus grave défaut apparaît dans le premier cas, les valeurs trouvées pour A deviennent beaucoup trop faibles et perdent plus ou moins leur signification brute.

Lor's d'une crue donnée, si l'on considère les valeurs de $A$ obtenues à la sortie des bassins les plus violemment arrosés et plus petits que 100 ou $50 \mathrm{~km}^{2}$, elles resteront lrès inférieures aux nombres trouvés pour les bassins compris entre 1000 et $5000 \mathrm{~km}^{2}$, peut-être $10000 \mathrm{~km}^{2}$. Parlout, en Europe Méditerranéenne et Centrale, en Californie, en Nouvelle-Angleterre, au Texas, dans toutes les manifestations de nubifragi, de Wolkenbrüche, de cloudbursts ou de pluies moins exorbitantes bien qu'encore remarquables par leur intensité, des faits multiples et concordants laissent pressentir une distorsion entre la puissance relative des débits maxima, d'une part, et le coefficient $A$, d'autre part. Deux exemples rendront éclatante cette contradiclion : la Talloria piémontaise, qui débouche dans le 'Tanaro moyen, au sud d'Asti, aurait débité $609 \mathrm{~m}^{3} / \mathrm{s}$ pour $33,1 \mathrm{~km}^{2}$ le 4 septembre 1948 , soit $18,4 \mathrm{~m}^{3} / \mathrm{s} / \mathrm{km}^{2}$, ce qui correspond à $\mathrm{A}=106$ seulement; pour les $4 \mathrm{~km}^{2}$ du Rhonnel à Clermontl'Hérault et $40 \mathrm{~m}^{3} / \mathrm{s} / \mathrm{km}^{2}$ en octobre 1860 , prétendus par certains peut-être sans exagération, A ne vaut que 80 . Il est cependant raisonnable de présumer qu'il s'agit de cas nombreux où les conditions naturelles présentent, de l'amont à l'aval, une homogénéité certaine et où la pluie a recouvert le bassin non avec une impossible idenlité, mais avec violence partout. Par comparaison avec ses valeurs pour l'Ardèche et l'Hérault moyens, A devrait valoir ici 140 ou plus pour bien caractériser la crue.

Un comportement inverse de A apparaît pour des valeurs-records dans le monde, lorsque la surface dépasse 5000 ou $10000 \mathrm{~km}^{2}$ et surlout $20000 \mathrm{~km}^{2}$ : en aucune région du monde, A n'a atteint, d'après les crues actuellement connues, les valeurs qu'on lui trouve pour des surfaces moindres; plus les bassins sont étendus, plus les records mondiaux de A éprouvent de la peine à égaler comme à dépasser 100 , sauf dans l'Asie des Moussons où A représente de 100 à 150 et plus pour 10000 à $50000 \mathrm{~km}^{2}$ sur de nombreuses rivières de l'Inde à cause de l'intensité durable et de l'ampleur spatiale des pluies; en Chine du Sud, à Wou-tchéou, pour 325000 $\mathrm{km}^{2} \mathrm{du}$ Si-Kiang, on donne 60000 à $70000 \mathrm{~m}^{3}$, même $71500 \mathrm{~m}^{3}$, en 1915 , soit 105 ou 125 pour $\mathrm{A}$, exception accentuée par l'étroitesse relative du lit majeur qui réduit les débordements, donc empêche les aplatissements dont bénéficie le Yang'T'sé Kiang, par exemple. Or, pour $1500000 \mathrm{~km}^{2}$ et plus, les crues les plus monstrueuses de l'Iénisséi et de la Léna, du Yang-Tsé Kiang, de l'Amazone ne peuvent guère se traduire par plus de 70 à 75 pour $A$. Quant au Congo, sa crue millénaire $\left(90000 \mathrm{~m}^{3} / \mathrm{s}\right.$ pour $\left.3700000 \mathrm{~km}^{2}\right)$ ne correspondrait qu'à $A=\mathbf{4 6 , 6 0}$.

Donc, si l'on pose en principe que A est fidè- 


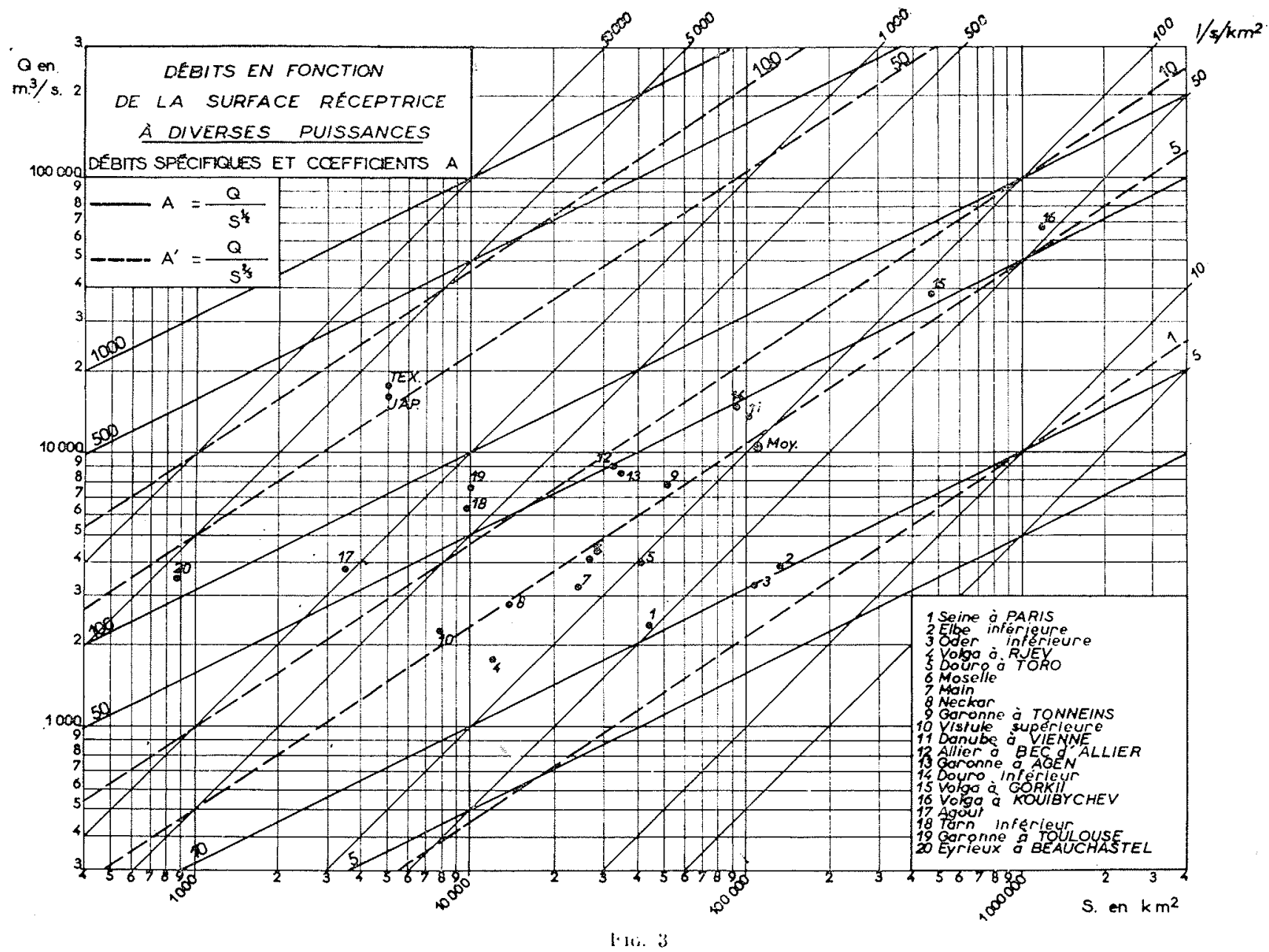

Valeurs de $A$ et de $A^{\prime}$, pour $n=1 / 2$ et $2 / 3$, respectivement.

lement représentatif pour les superficies petites, mais non minuscules, et moyennes, les coefficients numériques attribués, par la même formule où $S$ est affecté de l'exposant $1 / 2$, aux crues énormes seront trop faibles pour les bassins exigus et trop forts pour les vastes superficies. Des rectifications précises, dont les bases générales manquent, sont rendues difficiles parce que les valeurs de surfaces réceptrices à partir desquelles $\mathrm{A}$ devient sensiblement distordu varient selon les circonstances, selon les caractéristiques habituelles ou les anomalies des averses, selon les ruissellements locaux, à partir des centres violemment arrosés vers les débouchés, donc selon les conditions géophysiques: A se réduit beaucoup moins de l'amont à l'aval au-delà de 5000 ou de $100000 \mathrm{~km}^{2}$ dans l'Asie des Moussons qu'au Texas, par exemple, ce qui ne doit point surprendre puisque les recherches récentes soulignent la supériorité peu douteuse des précipitations que reçoit, en moins de 12 à 15 heures et surtout de 5 à 6 heures, la moitié sud-est des Etats-Unis sur les pluies les plus denses qui frappent l'Inde, éventuellement l'Indochine et la Chine, et sur les averses méditerranéennes les plus torrentielles.

\section{EMPLOI D'AUTRES EXPOSANTS}

Puisque l'exposant $1 / 2$ pour la surface réceptrice a comme conséquence la distorsion des valeurs de $A$, certains auteurs ont pensé pouvoir éliminer ce grave défaut, dans les divers cadres régionaux, en adoptant un exposant différent. D'où des formules du type $Q=A^{\prime} \cdot S^{n}$, dans les- 
quelles $n$ est compris, en particulier, entre $1 / 2$ et vaut $2 / 3$ ou $3 / 4$. Nous noterons en passant, avec surprise, cfue certains auteurs semblent envisager, pour leur formule, un coefficient mondial unique. Hypothèse absolument inacceptable, car ce serait prétendre ainsi que les équations traduisent les records connus ou possibles pour des surfaces de n'importe quelle étendue, indépendamment des facteurs géographiques et notamment des records possibles pour les averses.

Dans la présente étude, les valeurs expérimentales ou hypothétiques de $\mathrm{A}^{\prime}=\mathrm{Q} / \mathrm{S}^{2 / 3}$ ont été calculées pour tous les débits-records utilisés. Un résultal très appréciable a été obtenu : lors des crues de type très torrentiel et concentré (rivières cévenoles, petits cours d'eau des Etats-Unis en pleine saison chande...), les indications fournies par $A^{\prime}$ nous semblent plus conformes au classement, à l'appréciation en gravité relative des phénomènes naturels que ce n'était le cas pour les chiffres comparés de A. Par exemple, $\mathrm{A}^{\prime}$ vaut 63,6 pour le Rhonnel à Clermont-l'Hérault, 67,8 pour la Boyme tout près de là et 50 pour l'Ar- dèche à Aubenas, alors que A atleignait aux mêmes lieux respectivement 80,112 et 140 (soit presque une variation du simple au double). Mais pour des bassins plus grands, notamment pour plus de $100000 \mathrm{~km}^{2}$, sous les conditions géographiques les plus fréquentes en ce monde, $A^{\prime}$ diminue encore plus que $A$, ainsi que le monlre le tableau ci-dessous :

\begin{tabular}{|c|c|c|c|}
\hline \multicolumn{2}{|c|}{ Avec $\mathrm{A}=50$, nous obtiendrons : } & $\begin{array}{c}\text { ce qui } \\
\text { donnera } \\
\text { pour } \mathrm{A}^{\prime}\end{array}$ \\
\hline pour & $\mathrm{m} 3 / \mathrm{s}$ & $\mathrm{I} / \mathrm{s} / \mathrm{km}^{2}$ & \\
\hline & & & \\
$10000 \mathrm{~km}^{2}$. & 5000 & 500 & 10,8 \\
$20000 \mathrm{~km}^{2}$. & 7070 & 353 & 9,3 \\
$50000 \mathrm{~km}^{2}$. & 11180 & 223 & 8,1 \\
$100000 \mathrm{~km}^{2}$. & 15800 & 158 & 7,3 \\
2 millions km² & 70700 & 35,3 & 4,6 \\
\hline
\end{tabular}

Or, toutes ces valeurs sont, pour les débits, fort compatibles entre elles et tres admissibles

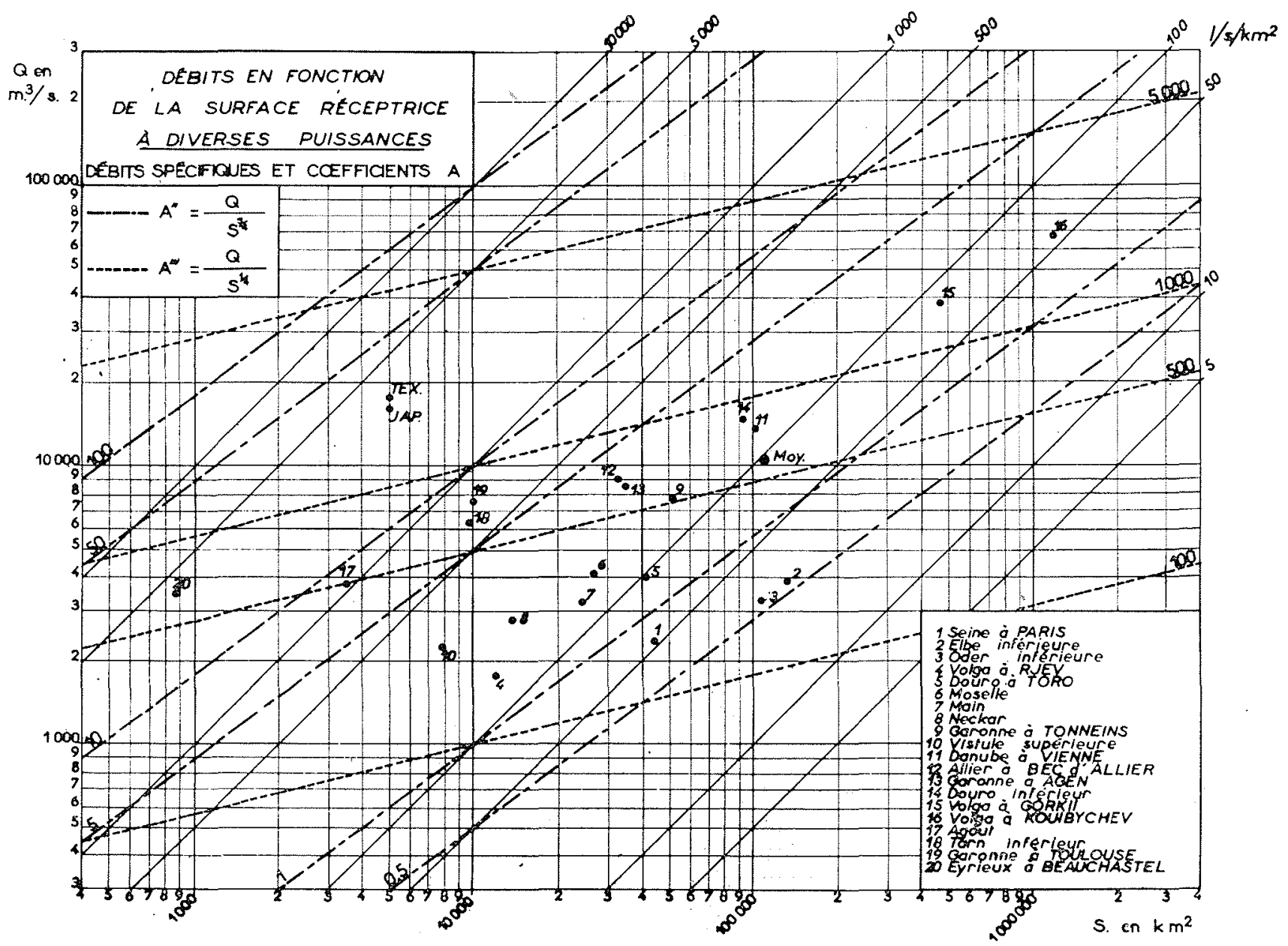

FIG. 4

Valeurs de $A^{\prime \prime}$ et de $A^{\prime \prime \prime}$, pour $n=3 / 4$ et $1 / 4$, respectivement. 
de l'amont à l'aval dans un bassin à réseau suffisamment convergent ou exposé à des concordances efficaces sans de très gros débordements qui aplatiraient les débits de pointe.

Comparons maintenant $A$ et $A^{\prime}$ appliquée à de grands bassins. Prenons $A^{\prime}=Q^{\prime} / S^{2 / 3}=15$.

Nous obtenons pour $10000 \mathrm{~km}^{2}$ :

$$
\begin{gathered}
\mathrm{Q}=6970 \mathrm{~m}^{3} / \mathrm{s}, q=697 \mathrm{l} / \mathrm{s} / \mathrm{km}^{2} \\
\text { et } \mathrm{A}=\mathrm{Q} / \mathrm{S}^{1 / 2}=69,7,
\end{gathered}
$$

crue très possible intégrée dans un phénomène hydrologique qui, pour $500000 \mathrm{~km}^{2}$, donnerait, avec $\mathrm{A}=69,7, \mathrm{Q}=49500 \mathrm{~m}^{3} / \mathrm{s}$ et $q=95 \mathrm{l} / \mathrm{s} / \mathrm{km}^{2}$ c'est-à-dire à peu près le maximum de l'Ohio en février 1937). Avec $A^{\prime}=15, Q=94500 \mathrm{~m}^{3} / \mathrm{s}$ et $q=189 \mathrm{l} / \mathrm{s} / \mathrm{km}^{2}$ (débits qui ne semblent guère réalisables), alors que, pour $10000 \mathrm{~km}^{2}, \mathrm{~A}^{\prime}$ indiquait un maximum très possible.

D'autres exemples. nous confirmeraient que, si A (fonction de $\mathrm{S}^{1 / 2}$ ) se traduit par des valeurs trop faibles quand il s'agit de très petits bassins, $\mathrm{A}^{\prime}$ (fonction de $\mathrm{S}^{2 / 3}$ ) n'est plus fidèlement représentatif quand il est appliqué à de très grands bassins. Le coefficient $A^{\prime \prime}$, fonction de $S^{3 / 4}$, serait-il plus satisfaisant? Le tableau de débits

\begin{tabular}{|c|c|c|c|c|c|}
\hline $\mathrm{km}^{2}$ & $\begin{array}{c}Q \\
\mathrm{~m}^{3} / \mathrm{s}\end{array}$ & $0 \frac{\stackrel{0}{\Xi}}{\frac{0}{3}}$ & 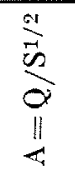 & 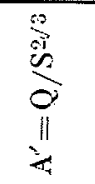 & 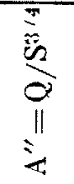 \\
\hline 10000 & 6000 & 600 & 60,0 & 12,90 & 6,00 \\
\hline 100000 & 20000 & 200 & 63,3 & 9,25 & $3, \overline{3} \cdot 4$ \\
\hline $500000 \ldots$ & 45000 & 90 & 63,6 & 7,15 & 2,39 \\
\hline $1000000 \ldots$ & 60000 & 60 & 60,0 & 6,00 & 1,90 \\
\hline
\end{tabular}
cohérents ci-dessous qui donne les chiffres des trois coefficients, révèle des écarts plus grands pour $A^{\prime \prime}$ que pour $A^{\prime}$, puisque les valeurs de $A^{\prime}$ sont dans le rapport de 2,15 à 1 tandis que celles de $A^{\prime \prime}$ sont dans le rapport de 3,15 à 1 .

Quels que soient les vices sérieux de ces coefficients, leur emploi pour les évaluations sommaires présente, outre l'avantage de la rapidité (qui peut avoir pour contrepartie une imprécision certaine), le grand mérite de varier beaucoup moins en fonction de surfaces réceptrices très changeantes que les débits spécifiques de pointe d'après lesquels on peut vouloir évaluer les débits bruts; la preuve en est apportée par le tableau précédent. Pratiquement, malgré les imperfections de ces coefficients, on aura intérêt, en de nombreux cas, à calculer a priori des débits de pointe avec $A$ plutôt qu'avec des débits spécifiques jugés convenables. Et, pour de très petites surfaces (moins de $500 \mathrm{~km}^{2}$ et surtout moins de $100 \mathrm{~km}^{2}$ ), on choisira, pour la même évaluation, I'indice $A^{\prime}$ que l'on sait meilleur. Les graphiques $\mathrm{Q}=\mathrm{A} \cdot \mathrm{S}^{n}$ ont été construits pour un nombre limité de points; mais, sur une population de 22 points, on constate que 18 d'entre eux se groupent en formant un nuage; les seuls cas aberrants, mais point du tout illogiques et correspondant à des débits aussi exacts que possible pour les fleuves soviétiques, sont les points 15 et 16 (Volga à Gorkii et à Kouybichev) et les points concernant le Japon, puis le Texas (en réalité, les coefficients A supérieurs à 200 et 250 , même 300 pour le Texas, ne sont pas aberrants pour ces deux derniers pays). Bien qu'elle soit déjà expressive, il est évident que cette représentation acquerrait une valeur plus grande avec un échantillonnage beaucoup plus complet et si l'on pouvait ne mettre en comparaison que des crues de même probabilité, avec des points de jaugeage bien répartis pour toutes les surfaces continentales considérées.

\section{EXAGERATIONS POSSIBLES PAR DES APPORTS FACTICES}

Après avoir recherché par des preuves numériques les qualités et les défauts des coefficients de ces fonctions exponentielles ou des exposants eux-mêmes, il est utile de rappeler que des erreurs sont à craindre dans l'appréciation des facteurs géophysiques dont dépendent les crues et de souligner que notre documentation relative aux dites causes souffre de très graves insuffisances en de vastes contrées. Et il importe tout autant d'évoquer quelques mécanismes spéciaux d'aggravation qui peuvent fausser les phénomènes fluviaux en lendant les débils supérieurs à ceux que produiraient les seuls processus logiques du ruisellement non perturbé, si l'on peut dire ainsi. Ce dernier résulterait des précipitations ou des fontes de neige. Parmi les mécanismes qualifiés de factices, nous distinguerons essentiellement:

- le renforcement des pointes de crues par rupture d'un barrage occasionnel;

- le renforcement par des débits solides; 
- le renforcement par déformation purement mécanique de l'onde.

Il faut compter avec les débâcles dues aux ruptures d'obstacles transversaux : barrages parfois monumentaux formés par des éboulis ou par des glissements de terrain ou par des avancées glaciaires ou embâcles dus aux accumulations de silaces fluviales. Des lacs temporaires se vident brusquement par les brèches et créent une crue factice exorbitante, telle celle qui eut lieu le 2 janvier 1915 sur le Colorado sud-pampéen et a pu atteindre vers le lieu de rupture un maximum de 80000 à $100000 \mathrm{~m}^{3} / \mathrm{s}$. Les ondes ainsi produites ont pour trait commun de s'aplatir heaucoup de l'amont vers l'aval.

Les transports solides (boues et sables fins ou moyens en suspension, matériaux de fond plus grossiers) peuvent, en s'ajoutant à la masse d'eau, donner un débit bien supérieur, en certaines rivières, à l'appoint liquuide seul. Le fleuve Jaune, par exemple, a roulé en aô̂t 1933, à l'orisine de son delta, une si abondante quantité de boues en suspension que son débit liquide se trouvait accru de quelque $30 \%$. Mais c'est sur le Rio Puerco, affluent du Rio Grande del Norte (frontière du Mexique et des Etats-Unis), qu'on a mesuré la plus grande charge en troubles connue de nous: $254 \mathrm{dm}^{3}$ contre $320 \mathrm{dm}^{3}$ d'eau; le débit de cette rivière, qui n'est pas un torrent de montagne, était done accru de $79,5 \%$. Dans les très petits bassins de montagne, les renforts solides peuvent augmenter les débits de pointe de $160 \%$ et plus.

Enfin, M. Pardé envisage l'hypothèse d'un renforcement factice par effet de rattrapage et de chevauchement; les débits de pointe, ou plutôt ceux plus rapides qui les précèdent immédiatement, tendraient à gagner de vitesse les débits croissants, mais moins rapides, qui les devancent, et à se superposer à eux; le volume d'eau croîtrait peu à peu vers l'aval, sans apports affluents nouveaux. Malgré l'absence de preuves directes certaines, la question est posée; en effet, des exemples de débits (qui ne sont pas des maxima) chevauchant des flots plus lents à l'aval sont connus : ce sont les murs d'eau (walls of water) par lesquels débutent quantité de crues foudroyantes dues à des cloudbursts, dans certains genres de lits fluviaux, notamment dans les lits à sec ou peu recouverts par le faible débit antérieur à la crise.

En tout cas, l'exagération facile du maximum par une débâcle ou par de gros transports solides ou par les deux mécanismes à la fois ne fait aucun doute. Il est indispensable que les spécialistes recherchent si le troisième genre de perturbation peut intervenir; l'incertitude la plus grave se rencontre dans les cas, réels ou imaginaires, où l'onde serait devenue plus acérée en cheminant vers l'aval par suite de rattrapages.

\section{CONCLUSION}

Nous pouvons, semble-l-il, asseoir nos jugements essentiels sur les formules qui prétendent lier les débits maxima des crues à une puissance, inférieure à l'unité, des surfaces réceptrices par l'intermédiaire d'un coefficient $\mathrm{A}_{n}$ bien déterminé. Nous doutons, même pour l'exposant, de l'existence d'une formule valable universellement, ne serait-ce qu'à cause des considérables variétés géographiques à la surface du globe. En outre, à ce manque d'uniformité physique mondiale s'ajoute l'absence d'homogénéité des débits-records quant à leurs fréquences; pour être parfaitement significatives et comparables entre elles, d'une région à l'autre ou à l'intérieur d'une même région, les valeurs jusqu'à présent observées devraient s'appliquer à des crues de même intervalle moyen; or, les débits maxima ici considérés valent pour des crues de fréquences très différentes; il s'ensuit que, dans ces conditions, nos comparaisons et nos con- clusions ne sont pas exemptes de toute critique. Néanmoins, on peut essayer de se rapprocher de l'idéal cherché en considérant diverses valeurs de A observées avec certitude, dans les dernières décennies ou les derniers siècles, sur certaines rivières, mais point sur d'autres, plus ou moins voisines et soumises selon toute vraisemblance aux mêmes influences géophysiques (précipitations possibles, reliefs, terrains, etc.). Par simple transposition, mais après une sérieuse étude comparée en climatologie et en potamologie, puis après construction d'hydrogrammes théoriques adéquats, on admettra, pour ces cours d'eau mal connus, la possibilité de crues exceptionnelles aussi puissantes que sur ceux pour lesquels on possède des données numériques sûres.

Quels que soient les reproches mérités par les formules examinées et le caractère peu homogène, donc peu exacts, des résultats chiffrés 
obtenus, on ne peut contester à ces derniers, même présentés entre des limites très distantes, une signification capitale. Les valeurs-types régionales judicieuses de $A_{n}$ permettent d'évaluer rapidement des ordres, souvent point absurdes, de grandeur à craindre pour les crues sur maintes rivières.

\section{I S CUSSION}

President : M. DEYMIŚ

M. le Président félicite M. Charter pour la clarté de son exposé et remercie M. Pardé d'avoir rassemblé cette documentation considérable sur les relations des débits de crues avec les superficies des bassins versants.

M. PARDÉ joint ses félicitations à celles adressées par M. le Président à M. Chanter. Il indique qu'indépendamment du mémoire succinct publié ci-dessus, il a rédigé, sur cette question, un très important document qui doit etre publié par ses amis les géographes espagnols et dont il remettra un exemplaire à la S.H.F.; entre temps, une copie du texts original de ce document est entre les mains de $M$. Chantrer ou de M. DE BEaUREGARD qui peuvent les prêter aux membres intéressés. Dans le même but, M. ParbÉ remettra prochainement à la S.H.F. un exemplaire rectifié de ce texte ainsi qu'un jen de 20 tableaux numériques où il a donné 700 ou 800 exemples pour une vingtaine de régions. Enfin, on peut consulter M. Pardé (1) et lui demander des détails sur l'exactitude plus ou moins approchée des chiffres.

M. Dumane, rappelant que M. Chartien a dit avoir tracé des droites correspondant à $n=0,25$, indique que la méthode des moindres carrés fait apparaitre un exposant $n=0,28$ avec la moindre dispersion pour les 22 cours d'eau dont $M$. Chartier a donné le tablcau : les coefficients A sont compris entre 1 et 4 au lieu de 1 et 10 . Il ajoute qu'il a remarque, d'autre part, une diminution de $A$ avec la superficie du bassin, tout au moins lorsque celle-ci dépasse $5000 \mathrm{~km}^{2}$ et trouvé par ajustement statistique $n=0,37$ en éliminant les deux points aberrants du Japon et du Texas.

M. DE Rovvine demande si la difficulté de trouver un exposant convenable à $S$, pour obtenir un coefficient A satisfaisant, ne tient pas à ce que, en considérant sculement $S$, on néglige la forme plus ou moins allongée du bassin qui ne doit pas manquer d'influer sur l'écolulement des pluies.

M. PARDÉ répond qu'il a envisagé cette cause - parmi bien d'autres - dans son ouvrage complet, qu'il vient de mentionner.

M. Robier indique que la forme du bassin intervient, mais que le coefficient $n$ présente malgré tout des valeurs assez variables pour des bassins de forme moyenne : dans l'Union Française, où l'on a des régimes très variés, $n$ devient très petit $(\leqslant 1 / 4)$ pour les régions arides; au contraire, dans les régions à cyclones, comme celles des Etats-Unis, $n$ devient supérieur à $1 / 2$.

M. le Président suggère d'essayer d'obtenit une dispersion moins grande par l'ajustement de la loi à des fonctions autres que les exponentielles : de telles fonctions devraient se rapprocher de $S^{1}$ pour les très petits bassins et devenir, soit asymptotiques, soit paraboliques pour des très grands bassins.

M. FERRY fait remarquer que la dispersion des points, répartis sur" graphique logarithmique, ne permet pas de

(1) Ecrire à M. Pardé, Professeur à la Factilté des Sciences de Grenoble, 6, boulevard Maréchal-Foch, à Grenoble. définir une courbe moyenne avec quelque précision. C'est pourcuoi on se limite à la consideration d'une droite de pente plus ou moins accusée, ce qui correspond a la formule en $S \alpha$.

M. Turc propose une formule à deux paramètres :

«En fait, dit-il, pour $S$ très petit, il faudrait $n \# 1$, en considérant une averse répartic uniformément à l'intérieur de la surface. Au contraire, le plafonnement de A lorsque $S$ devient grand suggère que l'exposant $1 / 2$ lui-même est alors trop grand.

"Il faudrait donc essayer une formule traduisant une diminution de l'exposant lorsque S croit; d'une façon ou de l'autre, cela revient à essayer une formule à deux paramètres, au lien du seul paramètre $n$; si l'on accepte cet inconvénient (deux paramètres au lieu d'un), on peut envisager :

$$
Q=A \frac{\mathrm{s}}{\alpha+\mathrm{S} \beta}
$$

on pourrait essayer $\alpha=100$ (si $\mathrm{S}$ est exprimé en $\mathrm{km}^{2}$ ) et $\beta=2 / 3$, comme valeurs des deux parametres.

«Il cst probable que l'introduction d'une telle formule laisserait encore subsister des difficultés importantes, comme l'a souligné M. Parbé. »

M. PaxpÉ pense qu'on pourrait en effet perfectionner la loi et il autorise les chercheurs à utiliser ses tableaux pour essayer d'y parvenir (2). Mais une formule unique parait difficile à trouver, en raison de l'extrême complexité des phénomènes intervenant dans la relation que l'on veut représenter (agencement des réseaux, décroissances exponentielles des pluies et des écoulements en fonction d'une pluie donnée). De ce fait, que la formule comporte un ou deux paramètres, il faudra affecter i ceux-ci des valeurs régionales ou sous-régionales par tatomnements, par connaissances, par raisonnements.

M. Trxeront donne dans le tableau ci-après les valeurs des crues décennales actuellement retenues en Tunisie, avec, en regard, leurs valeurs déduites de la formule $Q=30 \sqrt{\mathrm{S}}$

\begin{tabular}{|c|c|c|}
\hline $\begin{array}{c}\text { Surface des } \\
\text { bassins versants }\end{array}$ & Crues décennales & $30 \sqrt{\mathrm{S}}$ \\
$\mathrm{km}^{2}$ & $\mathrm{~m}^{3} / \mathrm{s}$ & $\mathrm{m}^{3} / \mathrm{s}$ \\
\hline 10000 & 2500 & 3000 \\
1000 & 1000 & 950 \\
100 & 300 & 300 \\
10 & 70 & 95 \\
1 & 20 & 30 \\
\hline
\end{tabular}

M. TIxenont ajoute :

«Il parait difficile de trouver une formule théorique-

(2) M. Parlé désire seulement qu'on le cite lorsqu'on se sert de ses documents. 
ment valable pour couvir tout le champ des superficies, car les facteurs principaux diffèrent. Pour les grands bassins, le débit des crues est surtout en rapport avec les fluctuations météorologiques. Pour les petits bassins, le débit des crues est surtout en rapport avec le temps de concentration des ruissellements et avec l'intensité des précipitations, telles qu'on peut les relever en un point donné avec un pluviomètre enregistreur.

«Le débit de $20 \mathrm{~m}^{3} / \mathrm{s}$ du tableau ci-dessus correspondrait au ruissellement total d'une précipitation d'intensité $72 \mathrm{~mm}$ à l'heure. On peut le considérer comme un maximum de la crue décennale, étant donné les statistiques de pluies enregistrées, qui donnent un chiffre inférieur pour les pluies d'une durée d'une demi-heure. »

M. le Président remercie les personnes qui sont intervenues dans la discussion et clôt celle-ci afin de respecter l'horaire.

M. PAnDÉ nous communique la note suivante qui lui a été remise par M. Coutagne à propos de sa communication :

« Je rappelle que la formule symbolique $O=A \sqrt{\mathrm{S}}$ peut être légitimée théoriquement par des considérations topographiques, pour des bassins homothétiques (voir ma communication de 1930 présentée a l'Association Internationale d'Hydrologie). Antérieurement, une telle formule avait été, je crois m'en souvenir, suggérée par un
Iydrologue anéricain. Plus genéralement, on a $Q=\mathrm{AS}^{n}$, n étant calculé par l'étude de la corrélation entre log 0 et $\log \mathrm{S}$ (linéaire). Un tableau concernant 14 régionstypes des Etats-Unis a été présenté il y a quelques années, $n$ étant égal généralement ì $1 / 2$, quelquefois à $2 / 3$.

« J'ai, ultérieurement, au $4^{\mathrm{e}}$ Congrès des Grands Barrages (1949) présenté quelques observations à ce sujet, illusirées par quelques exemples et figures. En particulier, j'indiquais pour l'Ardeche et les bassins voisins, $A=150$, chiffre confirmé par les crues cévenoles récentes, comme l'a indiqué M. Panoé dans son dernier mpport à ce sujet.

« Le coefficient $A$, de toute manière, ne constitue evidemment qu'un indice symbolique, d'une valeur toute relative, valable seulement pour les bassins d'une certaine étendue, pour caractériser l'intensité des crues possibles dans telle ou telle région particulière, tel on tel régime pluviométrique. La formule avec $A=44$ convient en particuliel pour la Columbia River considérée à différents points de son cours.

«Pour la Durance à Mirabeau, on a $A=40$. Personnellement, j'estime que ce coefficient ne doit pas dépasser 60 pour la rivière à Serre-Poncon, ce qui donnerait cnviron $1 \mathrm{~m}^{3}$ au $\mathrm{km}^{2}$, débit qu'on peut etre amené a majorer quelque peu, etant donné les risques d'un tel ouvrage.

A. Covative, 5 mars 1959 .

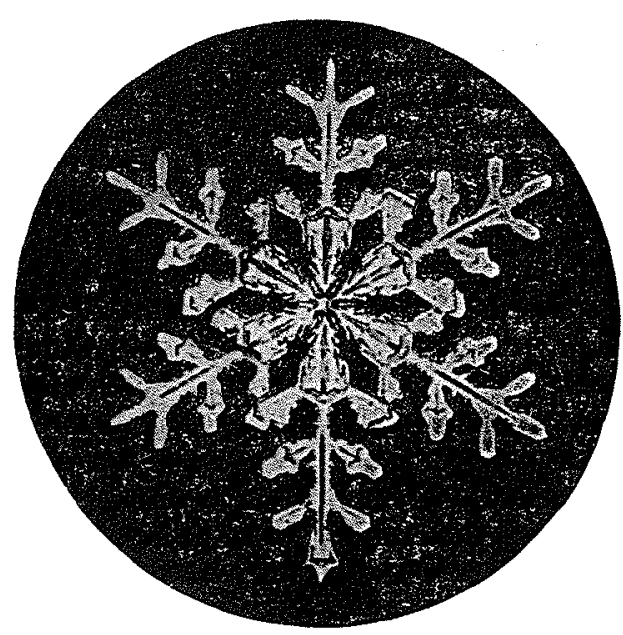

